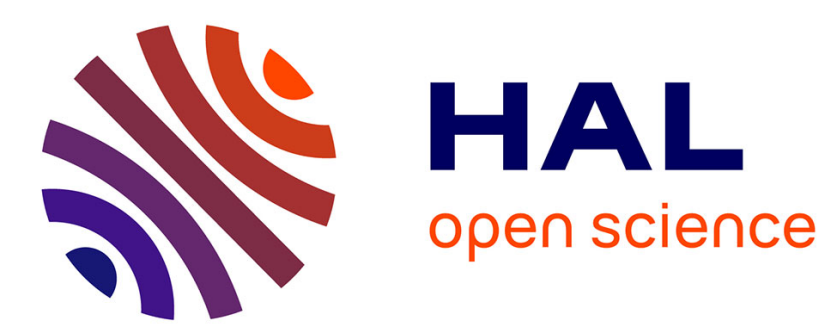

\title{
Design and experimental validation of a snapshot polarization contrast imager
}

Arnaud Bénière, Mehdi Alouini, François Goudail, Daniel Dolfi

\section{To cite this version:}

Arnaud Bénière, Mehdi Alouini, François Goudail, Daniel Dolfi. Design and experimental validation of a snapshot polarization contrast imager. Applied optics, 2009, 48 (30), pp.5764-5773. 10.1364/AO.48.005764. hal-00664889

\section{HAL Id: hal-00664889 \\ https://hal.science/hal-00664889}

Submitted on 6 Apr 2012

HAL is a multi-disciplinary open access archive for the deposit and dissemination of scientific research documents, whether they are published or not. The documents may come from teaching and research institutions in France or abroad, or from public or private research centers.
L'archive ouverte pluridisciplinaire HAL, est destinée au dépôt et à la diffusion de documents scientifiques de niveau recherche, publiés ou non, émanant des établissements d'enseignement et de recherche français ou étrangers, des laboratoires publics ou privés. 


\title{
Design and experimental validation of a snapshot polarization contrast imager
}

\author{
Arnaud Bénière, ${ }^{1,2, \star}$ Mehdi Alouini, ${ }^{2,3}$ François Goudail, ${ }^{1}$ and Daniel Dolfi' \\ 'Laboratoire Charles Fabry de l'Institut d'Optique, Centre National de la Recherche Scientifique, \\ Université Paris-Sud, Campus Polytechnique, RD 128, 91127 Palaiseau, France \\ ${ }^{2}$ Thales Research and Technology-France, 1 Avenue Augustin Fresnel, 91767 Palaiseau Cedex, France \\ ${ }^{3}$ Institut de physique de Rennes, Centre National de la Recherche Scientifique, \\ Université Rennes 1, 35042 Rennes, France \\ ${ }^{*}$ Corresponding author: arnaud.beniere @ institutoptique.fr
}

Received 3 April 2009; revised 13 July 2009; accepted 24 September 2009;

posted 25 September 2009 (Doc. ID 109610); published 14 October 2009

\begin{abstract}
We present a degree of polarization imaging system based on a Wollaston prism and a single CCD camera. This architecture eliminates technical inaccuracies and noise sources that are present in experimental setups containing a polarization switching element. After the acquisition of two images corresponding to two orthogonal states of polarization, one can compute the orthogonal state contrast image (OSCI), which is an estimate of the local degree of polarization of the backscattered light when the observed materials are purely depolarizing. The instrument design coupled to an efficient calibration enables the estimation of the OSCI from a single image acquisition and significant reduction of technical noise present in other polarization imaging systems. The setup was tested in realistic conditions where it represents a real asset. (C) 2009 Optical Society of America

OCIS codes: $\quad 260.5430,110.0110,120.5410$.
\end{abstract}

\section{Introduction}

Imaging systems that measure the degree of polarization (DOP) of light backscattered by a scene arouse great interest in several domains such as machine vision, biomedical imaging [1,2] $]$, and remote sensing [3]. They can, for example, reveal or increase contrast between regions of a scene that have similar intensity reflectivities but different polarimetric properties [4]. They are also able to improve the visibility through a diffusive medium [5-8]. In this paper we will consider active polarimetric imaging systems, which means that the observed scene is illuminated with a controlled state of polarization.

One of the simplest active polarimetric imaging principles consists of illuminating the scene with a totally polarized light beam and computing the orthogonal state contrast image (OSCI) from two inten-

0003-6935/09/305764-10\$15.00/0

(C) 2009 Optical Society of America sity images of the same scene. The first intensity image is formed with the fraction of the backscattered light polarized parallel to the incident light, and the second one with light polarized orthogonal to the incident one. It should be noted that the polarization state of the incident light need not be linear, but may be any pure polarization state on the Poincaré sphere. This simple approach is relevant in many remote sensing scenarios, since it is shown in Ref. [9] that the OSC is an estimate of the DOP of the backscattered light if the observed materials are purely depolarizing. These materials do not change the principal state of polarization of the incident light, which means that they do not present diattenuation or retardance.

Analysis along two orthogonal states of polarization is often achieved by switching a polarization sensitive device such as a rotating linear polarizer [10], a liquid crystal-based polarization rotator [4], a variable retarder $[11,12]$, or a ferroelectric modulator $[13,14]$. However, this type of approach has two 
drawbacks. The first one is that acquisitions of the two images are performed sequentially (one before and one after switching the element). For instance, the observed scene may change rapidly owing to the vibration of the carrier, the atmospheric turbulence, or the movement of a fast target. It thus appears differently in the two images, leading to erroneous estimation of the DOP, especially on the edges of the objects. The second drawback is that the polarization switching element may introduce perturbations, which may change with time or temperature and thus cannot be easily calibrated and corrected in a real field system. A solution to these problems is to use a polarizing cube beam splitter that separates the orthogonal linear states of polarization and forms the two images on two different detectors $[15,16]$.

To overcome these problems, we propose in this paper to design and test a system that is not suffering from noise due to polarization switching elements or multiple detectors by imaging simultaneously both orthogonal channels on the same detector. Such a device may have perturbations, but they are stable in time and can thus be calibrated and digitally corrected. Its estimation precision is then expected to be mainly affected by detector and photon shot noise, which is a fundamental limit. Of course there is a price to pay, which can be seen mainly as a loss in terms of spatial resolution or field of view. After reviewing the possible configurations for polarization separation in Section 2, we will dwell on the design of the architecture we have chosen in Section 3. We will then consider in Section 4 the residual optical aberrations of the setup and their correction, and we show that a calibration of such a system is easily performed. In Section 5 , we will quantify the precision of measurement of such a configuration. Section 6 will be devoted to experimental results and will highlight the advantages of simultaneous acquisition compared to the sequential mode in several situations.

\section{Choice of the Polarization Splitting Configuration}

In order to compute the OSCI, one needs to illuminate the scene with a totally polarized light beam and to acquire two intensity images: the first one, $X=\left\{X_{i}, i \in[1, N]\right\}$, is composed of $N$ pixels and is formed with the backscattered light in the same state of polarization as the incident light. The second one, $\boldsymbol{Y}=\left\{Y_{i}, i \in[1, N]\right\}$, is formed with the light polarized orthogonally to the incident state. For the sake of simplicity, we will use one-dimensional notation for images. The OSCI is therefore defined in the following way:

$$
\boldsymbol{P}=\left\{P_{i}, \in[1, N]\right\} \quad \text { with } P_{i}=\frac{X_{i}-Y_{i}}{X_{i}+Y_{i}} .
$$

This image represents the degree of polarization of the backscattered light if the observed materials only depolarize the incident light [9], which is a good approximation for outdoor scenes [4].
To form these two images on the same detector, a birefringent beam splitter separates the beam of backscattered light into two fully polarized beams with orthogonal linear polarizations. This separation of orthogonal states of polarization can be achieved spatially or angularly, with convergent or collimated light as illustrated in Fig. 1. On the one hand, the spatial separation uses a beam splitter such as a Savart polariscope, which will split a beam into two linearly polarized, parallel and laterally shifted beams (see Figs. 1(a) and 1(b)). On the other hand, the angular separation rests on splitting a beam into two deviated beams, thanks to a Wollaston prism, for example (see Figs. 1(c) and 1(d)). Let us analyze each configuration presented in Fig. 1 . The one represented in Fig. 1(a) relies on the use of a spatial beam splitter with convergent light. This setup has already been studied by Weijers in [17] with a modified Savart element composed of two crossed crystals and is shown to be limited by astigmatism, which could be corrected by the use of a cylindrical lens. However, this is a complex architecture, and a single block configuration would be preferable. In Fig. 1(b), spatial separation is performed on a collimated beam of light with a Savart plate, which leads to two spatially separated collimated beams. Two lenses are then needed to form both images on the detector, and the aperture of the system is limited by the aperture of those lenses, which is equal to the distance separating the collimated outputs. To our knowledge, there is no example of systems based on this configuration in the literature since it is the method that requires the highest birefringence and thickness for a given beam diameter. Angular separation performed under convergent light with a Wollaston prism in Fig. 1(c) has been studied and fully characterized in [18]: this architecture was shown to dramatically suffer from astigmatism, coma, and anamorphic distortion. In addition, the images are not in general formed on the same focal plane. Configuration in Fig. 1(d) rests on an angular splitting with collimated light. We choose this configuration because the incident plane wave front actually separates into two

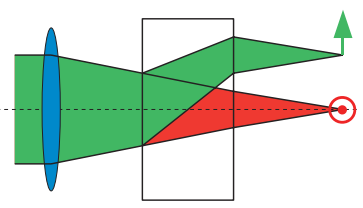

(a)

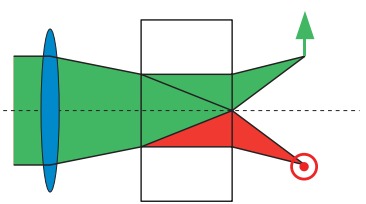

(c)

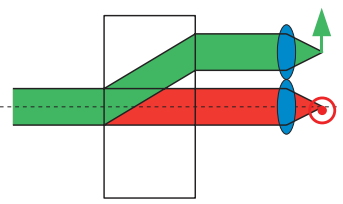

(b)

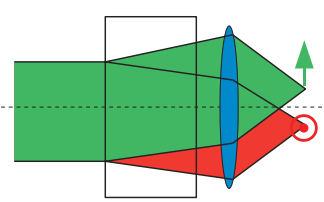

(d)
Fig. 1. (Color online) Schemes of (a), (b) spatial separation with a Savart polariscope and (c), (d) angular separation with a Wollaston prism associated with respectively convergent or collimated light. 
plane wave fronts, which does not introduce optical aberrations. This configuration was tested by Perrin et al. [19] in a passive polarimeter for astronomical applications. Harvey et al. [20] used this technique coupled to Lyot filters to obtain a snapshot spectral imager, but they did not exploit the polarization features of their system. The purpose of our system is to reveal contrast between objects with different polarimetric properties in a robust and easily implemented architecture.

In the above analysis, we have considered that the polarization state of illumination was linear, as well as the two states of analysis. The reason of this choice is purely technical: most beam splitters work with linear polarization. However, the proposed system can easily be adapted to illumination having any polarization state on the Poincaré sphere. One just needs to put a static phase retarder in front of the beam splitter in order to constitute polarization state analyzers parallel and orthogonal to any incident state of polarization. For example, a quarter wave plate will change the states of analysis from orthogonal linear to orthogonal circular states. One has to keep in mind, however, that in this case the admissible spectral width is likely to be limited by the phase retarder, which is not the case when performing analysis on orthogonal linear states of polarization.

\section{Basic Layout of Device}

The imaging system we have designed is based on the previously chosen configuration. The experimental setup is presented in Fig. 2(a). The lens L1 focuses the incoming light on an intermediate image. After collimation by lens L2, the beam is angularly separated from the quantity $\beta$ by the Wollaston prism. The ordinary and extraordinary beams are then focused on the CCD camera thanks to the lens L3, in order to form, respectively, images $\boldsymbol{X}$ and $\boldsymbol{Y}$. The rectangular mask $M$ is placed on the intermediate image in order to prevent the intensity images corresponding to the two polarizations from overlapping on the CCD camera.

The key component in this imaging system is the Wollaston prism. As shown in Fig. 2(b), a ray falling on the first prism with the incidence $i$ is slightly split because of the birefringence of the material. We simplify the notation, naming ordinary rays those that are ordinary in the first element of the Wollaston prism, and extraordinary those that are extraordinary in this element.

Outgoing light beams diverge from the prism, giving two linearly polarized beams, with the angle of divergence $\beta=D_{o}+D_{e}$, where $D_{o}$ and $D_{e}$ are, respectively, the ordinary and extraordinary deviation angles. These angles depend on the prisms' wedge angle $\alpha$, the wavelength of the light, the incidence angle $i$ on the Wollaston prism, and the ordinary and extraordinary refractive indices. Please note that the angle $i$ is oriented counterclockwise. By construction, it is seen that an incident monochromatic plane wave characterized by its wave front vector $\boldsymbol{k}$ will generate two plane wave fronts $\left(\boldsymbol{k}_{o}\right.$ and $\left.\boldsymbol{k}_{e}\right)$. As a consequence, this component does not strictly speaking introduce optical aberrations, and this is why we choose this type of beam splitter. However, the deviation angles $D_{o}$ and $D_{e}$ slightly depend on the incidence angle $i$, and so does $\beta$. This results in a vertical deformation of both ordinary and extraordinary images, which could be considered as a nonsymmetric anamorphic distortion.

For the design of the system, we will assume that the deviation of the Wollaston prism is independent

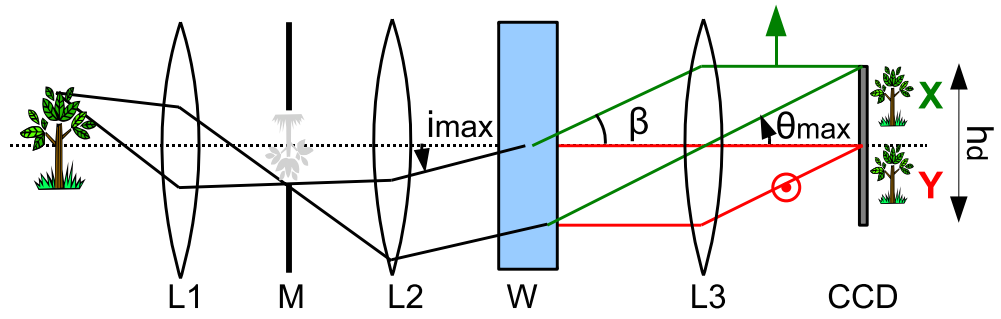

(a)

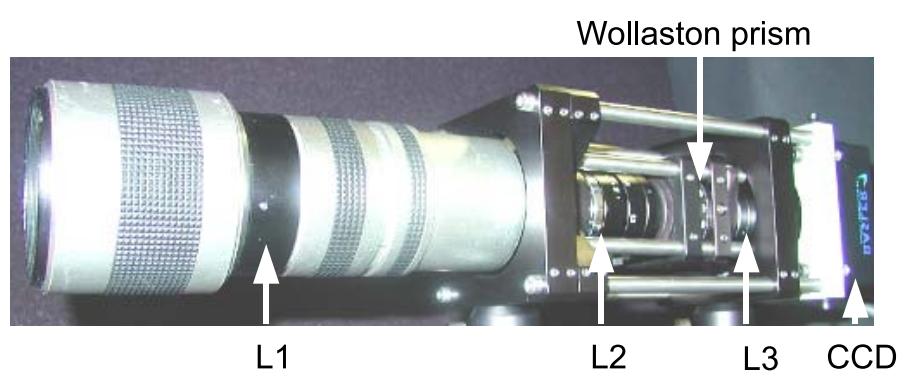

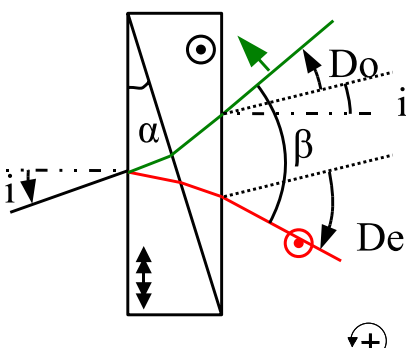

(b)

(c)

Fig. 2. (Color online) (a) Experimental setup composed of a Wollaston prism (W), three lenses (L1, L2, L3), a rectangular mask (M), and a CCD detector with a height of $h_{d}$. (b) Deviations in the Wollaston prism: an incident ray falls on the Wollaston prism with an angle $i$ and generates an ordinary ray and an extraordinary ray separated by the angle of divergence $\beta$. (c) Photograph of the experimental setup. 
of the incidence angle $i$ and that it can be approximated by [21]: $\beta \approx 2 \arctan (|\Delta n| \tan \alpha)$ with $\Delta n=$ $n_{e}-n_{o}$, where $n_{o}, n_{e}$ are the ordinary and extraordinary refractive indices. The system is designed so that images $\boldsymbol{X}$ and $\boldsymbol{Y}$ respectively cover half of the detector: the focal length of lens L3 is thus fixed. The height of the mask $M$ and the focal length of L2 are chosen to prevent images from overlapping on the detector. The field of view and the magnification of the system are then determined by the lens L1 and can be adapted to the targeted application.

Using these results, we built the experimental setup represented in Fig. 2(a). The detector is a Basler A312f, 12 bit CCD camera with a resolution of $782 \times$ 582 pixels and a pixel size of $8.3 \mu \mathrm{m} \times 8.3 \mu \mathrm{m}$. The Wollaston prism has a separation angle $\beta$ of $5^{\circ}$. As a consequence, the incidence $i$ will vary from $-2.5^{\circ}$ to $2.5^{\circ}$. The lenses L2 and L3 are imaging lenses with $35 \mathrm{~mm}$ focal length and $F / 2.1$ aperture. In our experiment, the lens L1 is a $28 \mathrm{~mm}-200 \mathrm{~mm}$ zoom. A photograph of the setup is presented in Fig. 2(c).

\section{Reduction of Residual Aberrations in Orthogonal State Contrast Images}

We have presented in the previous section the linear layout of the system whose aberrations are limited by construction. It provides a raw image with the parallel channel on the upper part of the detector and the orthogonal channel on the lower part, as shown in Fig. 3. Images $\boldsymbol{X}$ and $\boldsymbol{Y}$ are extracted from this raw image and need to be coregistered before computing the OSCI. The registration could be reduced to a simple translation if the images were not deformed, but we will see that this is not the case. Actually, the Wollaston prism and the lenses still introduce slight deformations that we address in this section. Two categories of aberrations must be considered: those that deteriorate images $\boldsymbol{X}$ and $\boldsymbol{Y}$ in

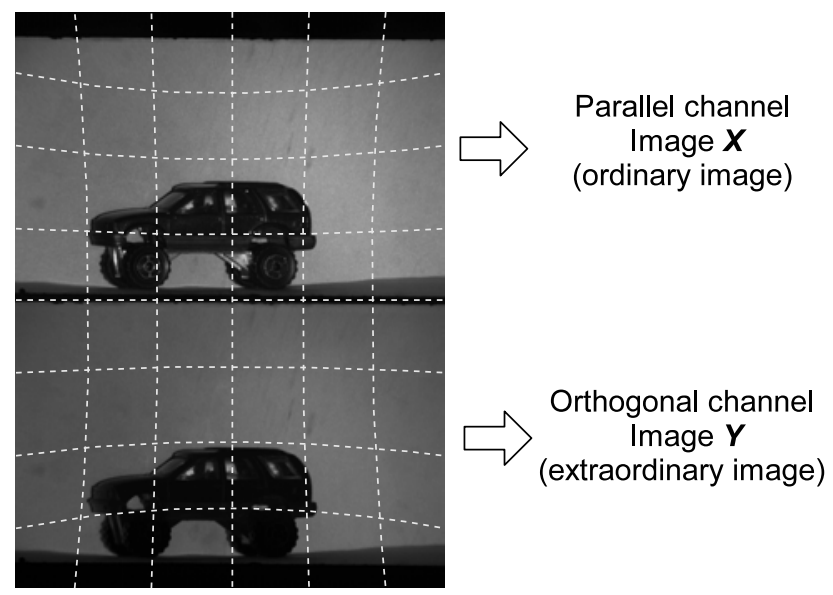

Fig. 3. (Color online) Raw image of a scene composed of a model scale car on a white diffusive background. The upper part is the ordinary image, which corresponds to the parallel state of polarization channel. The lower part is the extraordinary image, which corresponds to the orthogonal state of the polarization channel. A voluntarily exaggerated diagram of distortion introduced by L3 is superposed in dotted lines on the raw image. the same way, and those that do it in a different way. The first type mainly corresponds to some blurring of the images. These aberrations have been minimized by using off-the-shelf imaging achromatic doublets. Furthermore, we neglect the residual contribution, since it does not lead to perturbations specific to OSCI. The second type of aberrations can significantly deteriorate the quality of OSCI estimation since OSCI is based on a difference and a ratio of images as shown in Eq. (1). In this equation, pixels $X_{i}$ and $Y_{i}$ must refer to the same region in the scene for $P_{i}$ to be meaningful. OSCI is thus very sensitive to deformation of image $\boldsymbol{X}$ with respect to image $\boldsymbol{Y}$ when large spatial gradients are present in intensity or polarization. We will thus focus in this section on the second type of distortion-like aberrations, which lead to registration issues but can be corrected digitally.

\section{A. Nonsymmetric Anamorphic Distortion of the Wollaston Prism}

In order to characterize the aberrations of the Wollaston prism, we have represented in Fig. 4 the simulated evolution of the deviations $D_{e}$ and $D_{o}$ as a function of the incidence. These curves depend on the wavelength. In this paper we consider active systems in which the illumination is assumed monochromatic, and we will thus not address the problem of chromatism. In the following experiments, we use an interferometric filter centered on $632 \mathrm{~nm}$ with $10 \mathrm{~nm}$ full width at half-maximum in front of the illumination. However, multispectral configurations are conceivable if an appropriate calibration is used. One notices that both deviations vary conversely and nonsymmetrically: $\left|D_{o}\right|$ varies in a larger range than $\left|D_{e}\right|$. This implies that intensity images $\boldsymbol{X}$ and $\boldsymbol{Y}$ are not deformed in the same way. The height of image $X$ will be smaller than it should be, whereas the height of image $\boldsymbol{Y}$ will be larger. One can quantify the deformation introduced in our experimental setup. Let us
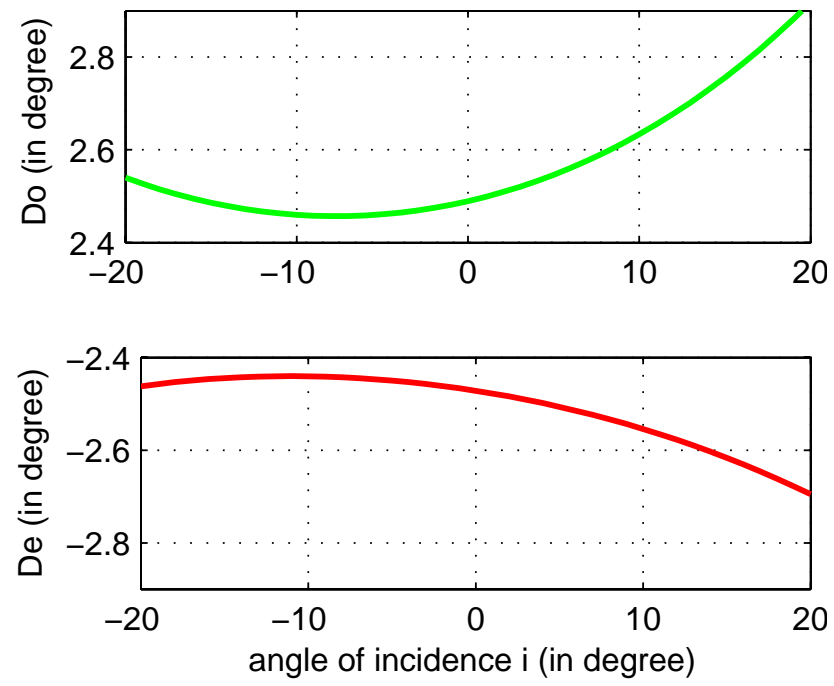

Fig. 4. Ordinary $\left(D_{o}\right)$ and extraordinary $\left(D_{e}\right)$ deviations of a $5^{\circ}$ separation Wollaston prism as a function of the angle of incidence $i$ for $\lambda=632 \mathrm{~nm}$. All angles are in degrees. 
assume that the images are registered using a translation only, with the reference on the center of the images $\left(i=0^{\circ}\right)$. In other words, image $\boldsymbol{Y}$ is translated so that its center is registered with the center of image $X$. The maximum shift between the images is thus reached on the edge of the images $\left(i=-2.5^{\circ}\right.$ or $i=2.5^{\circ}$ ) and is about $0.05^{\circ}$, which corresponds to registration mismatch of approximatively 4 pixels in our case.

As shown in Fig. 4, the minima of deviations are not obtained for a normal incidence but for $i=i_{\text {mde }} \approx$ $-8^{\circ}$ for the extraordinary beam and $i=i_{m d o} \approx-10^{\circ}$ for the ordinary beam. If the Wollaston prism is tilted of the quantity $i_{m d}=\left(i_{m d e}+i_{m d o}\right) / 2$, the deviations $D_{o}$ and $D_{e}$ will be close to their minimum and thus will vary less with incidence. The deformation of the images will be significantly reduced. With this modification, if the images are registered using a translation only, the maximum shift becomes less than 1 pixel. For better accuracy, as we know the deformation induced by the Wollaston prism, it is possible to correct it by software. However, we will show in the following section that the global system is affected by another deformation and that a global correction is required.

\section{B. Nonsymmetric Distortion Introduced by Lenses}

Optical aberrations introduced by lenses L1 and L2 are not so disturbing as they affect images $\boldsymbol{X}$ and $\boldsymbol{Y}$ the same way: they do not lead to misregistration. This is not the case for lens L3, which is positioned after the splitting element. It is seen in Fig. 3 that the distortion of lens L3 does not deform symmetrically images $\boldsymbol{X}$ and $\boldsymbol{Y}$. The top of image $\boldsymbol{X}$ is more distorted than the bottom, whereas it is the contrary for image $\boldsymbol{Y}$. Instead of focusing on each deforming elements of the system, we have chosen to perform an optical calibration of the whole system (that is, the
Wollaston prism and the lens L3). After the extraction from the raw acquisition, the values $X_{i}$ and $Y_{i}$ do not necessarily correspond to the same region in the scene. The image $\boldsymbol{Y}$ will be deformed into $\boldsymbol{Y}^{\prime}$ so that for each pixel, $X_{i}$ and $Y_{i}^{\prime}$ are the images of the same region in the scene.

For this, we used a two-dimensional polynomial transformation of order 4. To estimate the 30 unknown coefficients of this polynomial, one acquires the raw image of a grid of 170 small white dots on a uniform black background. A strongly depolarized illumination is used, in order to obtain nearly the same amount of light in images $\boldsymbol{X}$ and $\boldsymbol{Y}$. The barycenters of the dots are the handles that control the deformation. The polynomial transformation is inferred in the least square sense from the control points and used with a subpixel bicubic interpolation scheme to deform the whole image $\boldsymbol{Y}$.

As a means to pinpoint the efficiency of this method, we used the image of a chart of concentric black and white disks [see Fig. 5(a)]. We compute the OSC image. If the images were deformed in the same way, a simple translation would be sufficient to obtain $(X-Y)_{i} \approx 0, \forall i \in[1, N]$, but it is not the case. Indeed, if image $Y$ is translated so that its center is registered with that of image $X$, it is seen in Fig. 5(b) that the vertical shift between images $\boldsymbol{X}$ and $\boldsymbol{Y}$ increases with the distance from the center. We then apply the function $f$ obtained from the above described calibration. This procedure results in a much better registration [see Fig. 5(c)]. Actually we notice in the zoomed images of Figs. 5(d) and 5(e) that the registration precision is better than a pixel. Moreover, it is seen in the histograms represented in Figs. 5(f) and 5(g) that the polynomial registration gets rid of the erroneous absolute OSC values greater that 0.2 . The residual variance is due to photon and detector

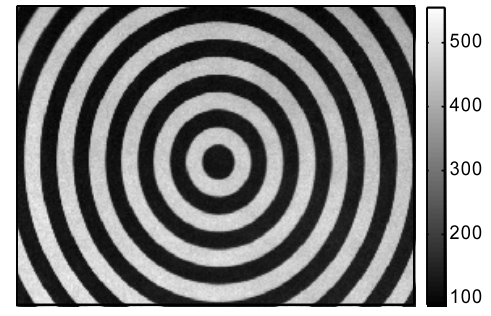

(a)

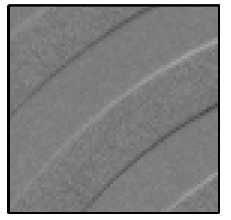

(d)

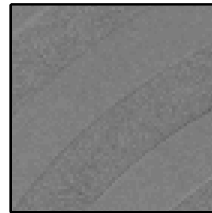

(e)

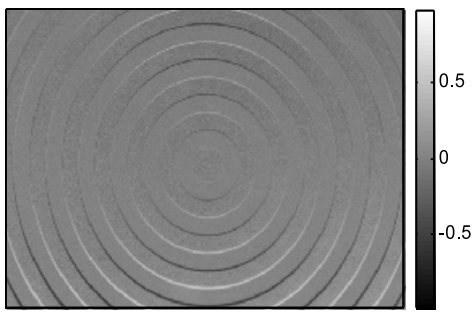

(b)

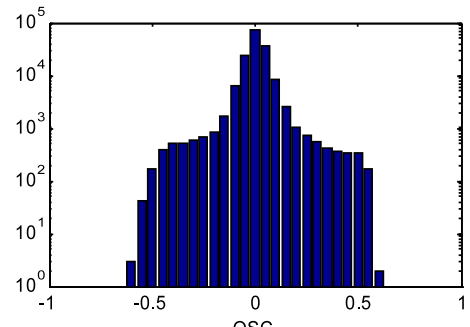

(f)

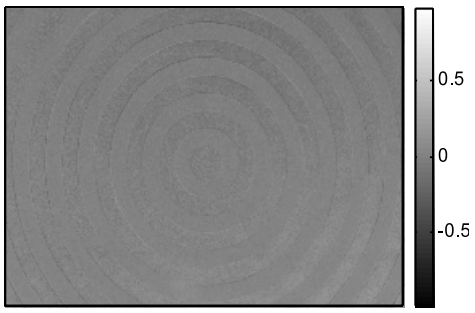

(c)

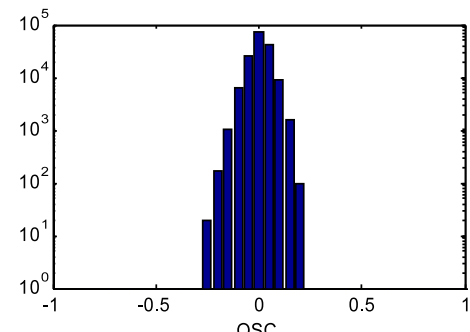

(g)

Fig. 5. (a) Intensity image of the chart composed of black and white rings. OSC images, if images $\boldsymbol{X}$ and $\boldsymbol{Y}$ are registered using (b), (d) a translation only or (c), (e) a polynomial transformation . (f), (g) Corresponding histogram in log scale. 
noise in images $\boldsymbol{X}$ and $\boldsymbol{Y}$. It is worth mentioning that on this image the rings are still discernible because of the photon noise that is more important in white rings, that is, where the backscattered intensity is higher.

It is seen that the registration procedure developed in this section shows performances better than a pixel. However, the residual error could be a problem at image edges if we consider high signal to noise ratio situations. It must be kept in mind that the registration procedure can be improved by increasing the number of control handles during the calibration. Furthermore, edge detection is standard operation in image processing and can easily be implemented to locate and discard regions where the OSC estimation is less precise.

\section{Polarimetric Calibration and Precision of Measurement}

The OSC imaging system designed in the previous system needs a polarimetric calibration to evaluate measurement errors. The image overlay procedure presented in the previous section is systematically applied. In order to obtain a uniform scene we use an integrating sphere with a hole of $5 \mathrm{~cm}$ diameter covered by an interferometric filter centered on $632 \mathrm{~nm}$ with $10 \mathrm{~nm}$ full width at half-maximum.

The first feature we want to measure is the polarization induced by the system. For this we focus our polarimetric imager on the filter, and we thus obtain intensity and OSC images of a depolarized uniformly illuminated scene. We estimate the mean and the variance in both images in a region of $50 \times 50$ pixels. The results are presented in Fig. 6 .

It is seen in Fig. 6(a) that the mean of the estimated intensity is proportional to the integration time, which proves the linearity of the detector. The estimated variance of the intensity also linearly

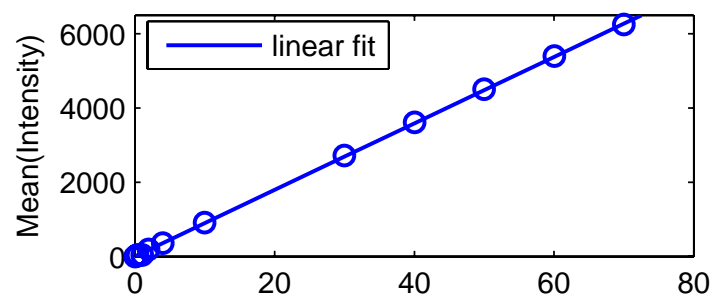

(a) Integration time in $\mathrm{ms}$

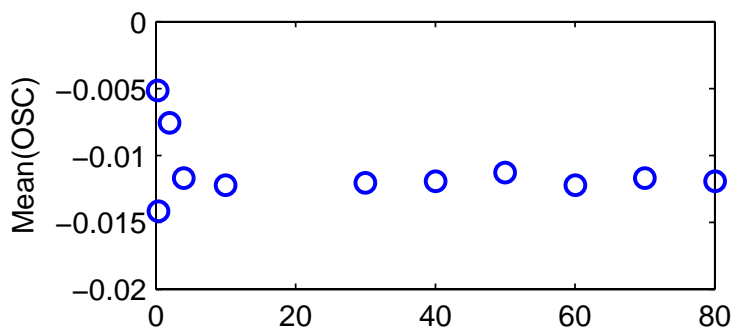

(c) Integration time in $\mathrm{ms}$ depends on the integration time [see Fig. 6(b)]: this justifies that, when the signal is sufficient, the system is limited by photon noise that can be modeled with a Poisson random variable.

Figure 6(c) shows that even if the light coming in the system is totally depolarized, the system measures an averaged OSC of approximately $P_{0}=$ -0.012 . We have checked that this phenomenon is not due to some residual polarization of the light coming out of the integrating sphere. Indeed, when the imaging system is rotated along its optical axis, we still measure an averaged OSC of -0.012 . This is because the Wollaston prism does not have exactly the same transmittance for both states of polarization. This systematic bias is corrected by multiplying image $\boldsymbol{X}$ by the constant $\eta_{0}=\left(1-P_{0}\right) /\left(1+P_{0}\right)=1.024$.

The second feature that must be evaluated is called the instrumental depolarization [22]. For this, we add a linear polarizer whose extinction ratio is 1:10000 on a rotating mount just after the interferometric filter. The polarimetric imager is focused on the polarizer, and we thus obtain intensity and OSC images of a totally polarized (DOP $=99.99 \%$ ), uniformly illuminated scene. The polarizer is oriented to maximize the intensity in image $\boldsymbol{X}$ and to minimize it in image $\boldsymbol{Y}$. If the beam splitter were perfect, we would measure an OSC of $99.99 \%$. This is not the case, as it is seen in Fig. 7: actually the mean OSC value is $P_{1}=98.2 \%$ (close to the value found in [22]) and does not depend on the integration time.

Concerning the precision of the computed OSC, we plot in Figs. 6(d) and 7(d) the variance of the OSC and the associated Cramer-Rao lower bound (CRLB) [23]. Let us recall that the CRLB is a lower bound on the estimation variance that can be reached by an unbiased estimator, and its derivation under photon noise is detailed in [24]:

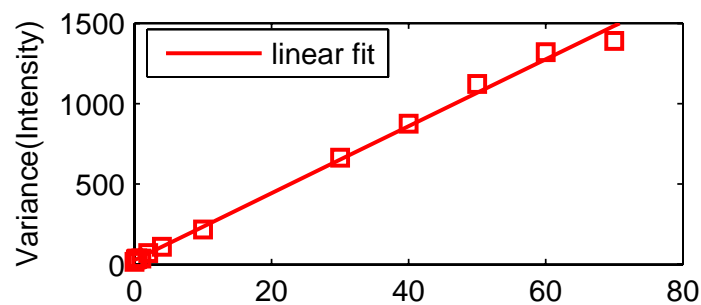

(b) Integration time in ms)

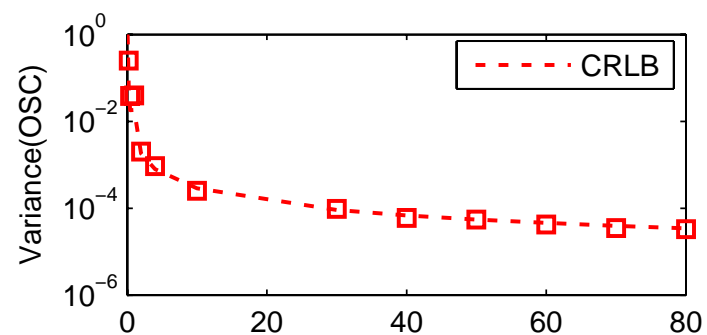

(d) Integration time in ms

Fig. 6. (Color online) (a) Mean and (b) variance of the intensity image estimated on a $50 \times 50$ pixels region when observing a totally depolarized light source. (c) Mean and (d) variance of the OSC. 


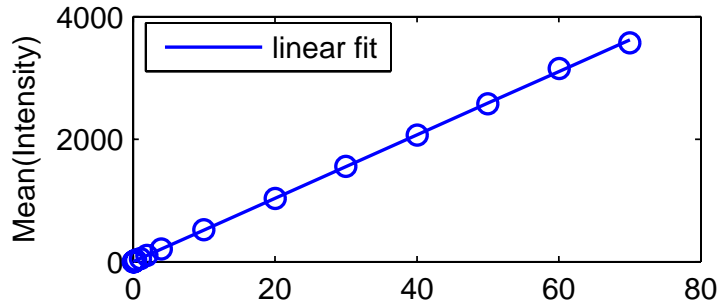

(a) Integration time in ms

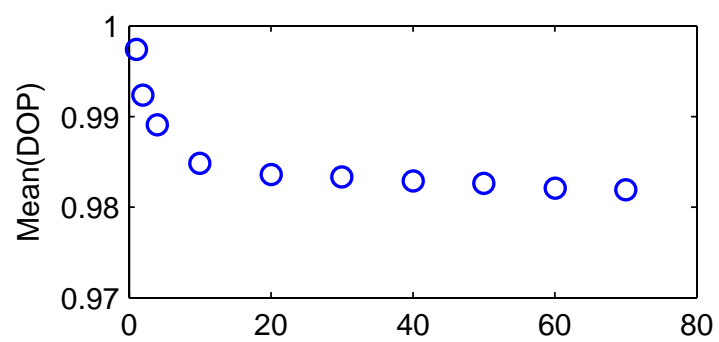

(c) Integration time in ms

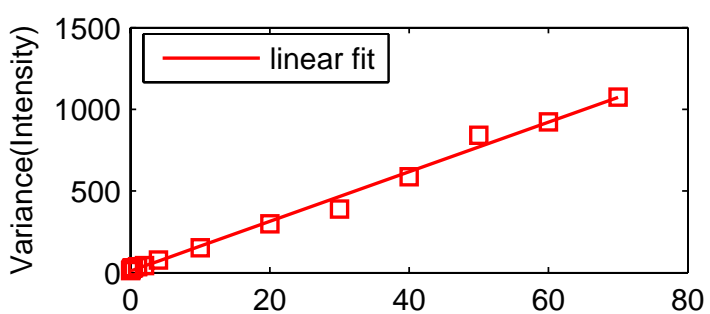

(b) Integration time in ms

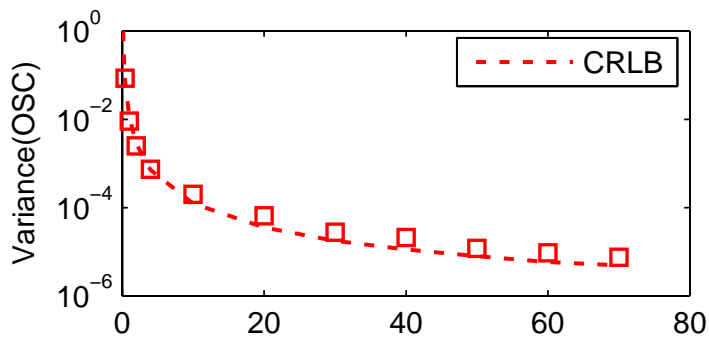

(d) Integration time in $\mathrm{ms}$

Fig. 7. (Color online) (a) Mean and (b) variance of the intensity image estimated on a $50 \times 50$ pixels region when observing a linearly polarized light source. (c) Mean and (d) variance of the OSC.

$$
\operatorname{CRLB}_{P}(g)=\frac{\left(1-P^{2}\right)}{q I}+\frac{2 g\left(1+P^{2}\right)}{(q I)^{2}}
$$

where $I$ is the intensity in gray value, $q=4.85$ is the number of photoelectrons per gray level, and $g$ is the passive contribution estimated at 100 photoelectrons. It has been shown that the parameter $g$ can account for detector noise or residual ambient light. In our model, the residual error after the flat-fielding of the sensor (compensation of the fluctuation of offset and gain of pixels) is also considered as detector noise and included in the parameter $g$. The estimated variance is very close to the CRLB, which justifies that the intensity images are mainly limited by photon noise and detection noise in homogeneous regions. Actually, it is seen in Fig. 5(e) that the error due to the overlay procedure is limited in the presence of strong gradients. In conclusion, our system presents slight biases due to instrumental polarization and depolarization. However, the targeted application is to reveal contrasts between objects of interest and a background rather than measuring the absolute value of the OSC. In such applications, the most important parameter is the estimation variance of OSC, and we have shown that it reaches its fundamental limit since it is very close to the CRLB.

\section{Validation of Concept on Some Real Situations}

We designed a simultaneous acquisition OSC imaging system coupled to an efficient calibration. This setup enables us to acquire OSC images free of technical noise, that is, perturbed only by detection noise or photon noise. It has been used in our recent studies [24-26] to validate estimation and detection algorithms on OSC images. In this section we will show how this system enables a good OSC estimation in real conditions. In particular, we will carry out experiments in situations where systems based on sequential acquisition $[3,14,27]$ show weakness and imprecision. The system designed in this paper can also be operated in a sequential acquisition mode to show the benefit of simultaneous acquisition: one just has to consider the parallel image $X$ in an acquisition and the orthogonal image $\boldsymbol{Y}$ in the subsequent acquisition. The distortion correction procedure is still applied but would not be required in a true sequential mode configuration (using a liquid crystal, for instance). However, the artifacts due to distortion correction are negligible in comparison to those induced by turbulence. The result is thus a reliable illustration of improvement obtained by performing simultaneous acquisition of two images.

\section{A. Imaging Moving Scenes}

When imaging a dynamic scene due to moving objects or to a displacement of the imaging system, it is easily understood that simultaneous acquisition is required. Actually, in the sequential mode with a dynamic scene, a pixel in image $X$ will not correspond to the same region as a pixel in image $\boldsymbol{Y}$.

Simultaneous acquisition is also required when imaging through turbulent media (like atmosphere), which often happens in outdoor remote sensing. Turbulence induces index fluctuations that result in random deviations of amplitude and phase of the light coming from the object. The further the observed object, the more the turbulence deforms the wave front coming from the scene. It is understood that if images $\boldsymbol{X}$ and $\boldsymbol{Y}$ are acquired sequentially, they are not perturbed in the same way because the turbulence evolves during and between the acquisitions. As an illustration, in Fig. 8, a small piece of bare metal is placed on a diffusive background with a camouflage pattern, so that it is not detectable on the intensity image Fig. 8(a). A hotplate placed between this scene and the imaging system creates turbulences. The intensity image Fig. 8(a) is thus slightly blurred 


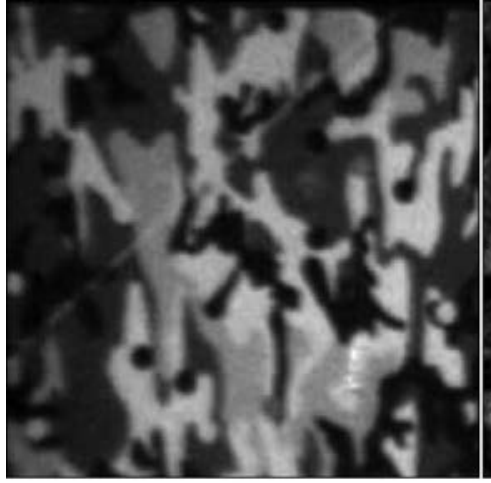

(a)

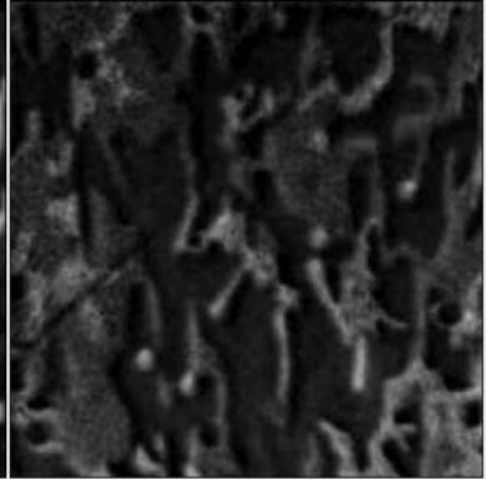

(b)

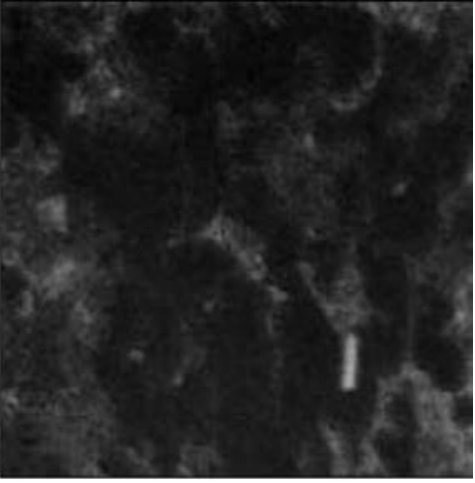

(c)

Fig. 8. $100 \times 100$ pixel image of a piece of bare metal on a diffusive camouflage pattern. (a) Intensity image and (b) sequential and (c) simultaneous OSCI with turbulences.

and deformed. In the OSCI with sequential acquisition Fig. 8(b), images $\boldsymbol{X}$ and $\boldsymbol{Y}$ are not affected in the same way by the turbulence and the small piece of metal disappears among artifacts due to the respective shift of spatial intensity gradients in images $\boldsymbol{X}$ and $\boldsymbol{Y}$.

When using simultaneous acquisition [see Fig. 8(c)], the OSC image is still deformed by the turbulence, but artifacts are not a matter of concern. In this case, the orthogonal image is deformed in the same way as the parallel image, so that the pixel $Y_{i}$ corresponds to the same white region.

One also notices smooth variations of the OSC of the background in Fig. 8(c). Regions that are dark in the intensity image appear a little brighter in the OSCI of Fig. 8(c). This phenomenon can be explained as in $[28,29]$ thanks to the Kubelka-Munk theory. Indeed, the light backscattered by an absorbing material mainly comes from surface scattering, which is weakly depolarizing. Dark regions thus have higher OSC. On the other hand, reflection on a white surface has a strong volume scattering contribution, which depolarizes more. A white region thus has a lower OSC.

\section{B. Pulse to Pulse Fluctuations}

In order to increase the range of remote sensing systems, pulsed light sources are classically used $[14,30]$. However, such sources are often affected by pulse-topulse intensity fluctuations. If we use a sequential acquisition, this means that images $\boldsymbol{X}$ and $\boldsymbol{Y}$ are not acquired with the same intensity, which results in errors of estimation of the OSC unless the pulse energy is recorded to perform a postcorrection.

Let us consider the case of sequential acquisition of a motionless scene with a pulsed and spatially uniform light source. We assume that the parallel image $X^{t}$ is acquired at time $t$ with the illumination $F^{t}$ and that the orthogonal image $\boldsymbol{Y}^{t+1}$ is acquired at time $t+$ 1 with illumination $F^{t+1}$. The reflectivity of the scene is time independent, that is, $\forall t, \forall i, X_{i}^{t}=F^{t} X_{i}$, and $Y_{i}^{t}=F^{t} Y_{i}$, with $X_{i}$ and $Y_{i}$ independent of $t$. The estimated OSC at pixel $i$ is expressed as follows:

$$
\hat{P}_{i}^{t}=\frac{F^{t} X_{i}^{t}-F^{t+1} Y_{i}^{t+1}}{F_{t} X_{i}^{t}+F^{t+1} Y_{i}^{t+1}}=\frac{P_{i}+\alpha^{t}}{\alpha^{t} P_{i}+1},
$$

where $P_{i}=\left(X_{i}-Y_{i}\right) /\left(X_{i}+Y_{i}\right)$ is the true OSC image and $\alpha^{t}=\left(F^{t}-F^{t+1}\right) /\left(F^{t}+F^{t+1}\right)$ represents the relative pulse fluctuation. If $\forall t, \alpha^{t}=0$, the pulse intensity is time independent, and the estimated OSC image corresponds to the true one. The evolution of the estimated OSC $\hat{P}_{i}^{t}$ as a function of $\alpha^{t}$ is plotted in Fig. 9 for different values of the OSC $P$.

In order to illustrate the error that can be obtained with such a setup, we used a scene composed of an optical chopper placed on a diffusive black background. The experiment is designed in such a way that there is weak contrast between the background (seen through the slots) and the chopper itself on the intensity image. The scene is illuminated with a red modulated high power light emitting diode (LED) to simulate pulse intensity fluctuations that are represented in Fig. 10(d). For purpose of comparison, we display OSCI images obtained with sequential acquisition [see Fig. 10(a)] and simultaneous acquisition [see Fig. 10(b)]. For each OSCI, a spatial average of the OSC is performed on 100 pixels corresponding

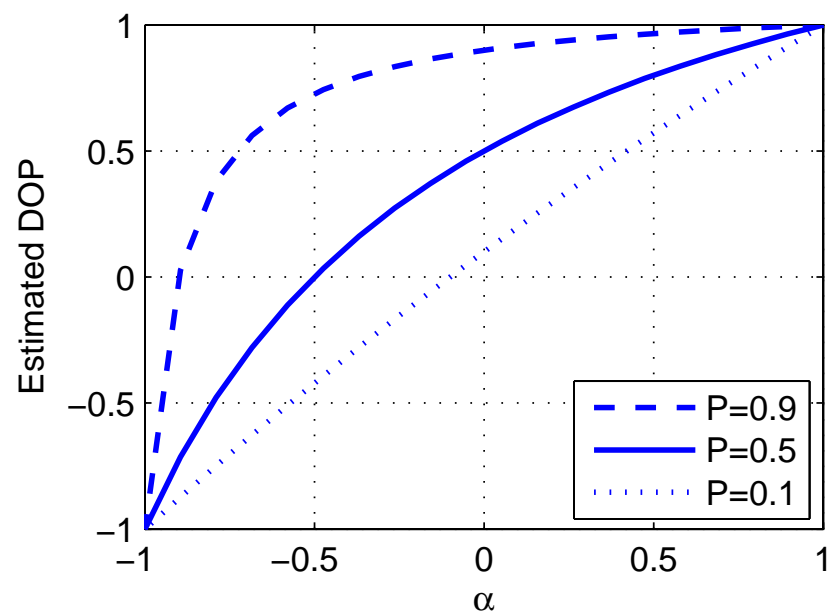

Fig. 9. (Color online) Estimated OSC as a function of the parameter $\alpha^{t}$, for different true value of the OSC. 


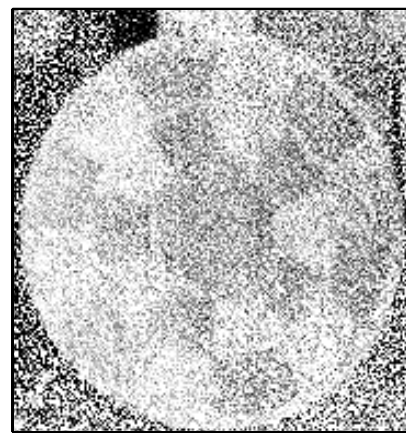

(a)

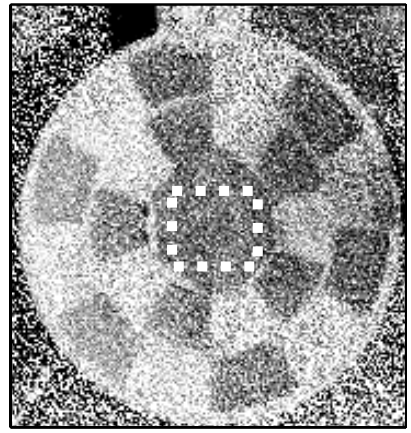

(b)

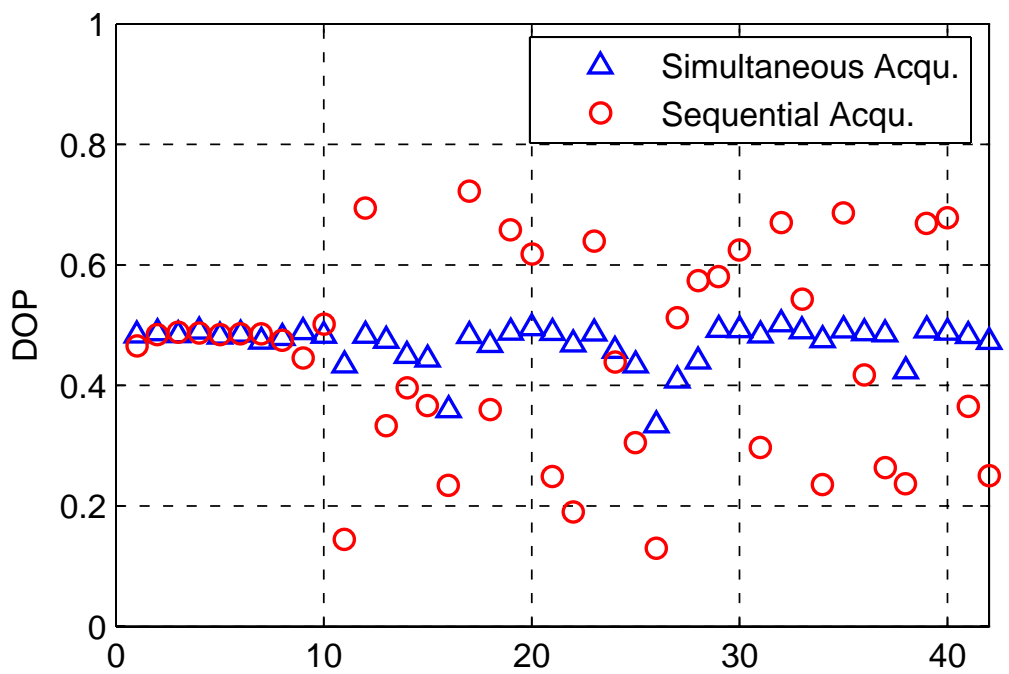

(c)

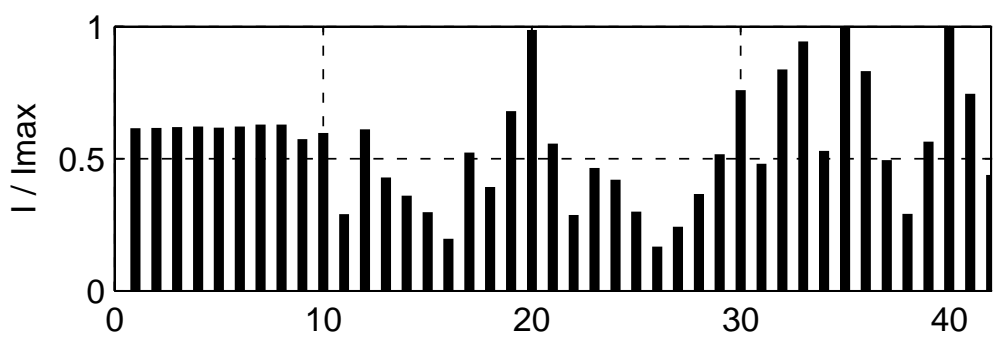

(d)

Fig. 10. (Color online) OSC images obtained with (a) sequential acquisition and (b) simultaneous acquisition with a pulsed light source. (c) For each acquisition the spatial mean of the OSC estimated on the black plastic delimited by dotted rectangle is compared for both acquisition modes. (d) Pulse to pulse fluctuations. The case $\alpha^{t}=0$ corresponds to the estimation of the true value of the OSC.

to the black diffusive plastic, and its evolution in time is plotted in Fig. 10(c). The averaged region is delimited in Fig. 10(b) by a dotted rectangle. With sequential mode, one pulse is used to acquire the parallel state image $X$, and the next one is used to acquire the orthogonal state image $\boldsymbol{Y}$. This leads to a severe loss of contrast and erroneous estimated OSC values. We assume in Fig. 10(d) that the intensity of a pulse does not exceed double the intensity of the previous, that is, $-1 / 3 \leq \alpha \leq 1 / 3$. This inequality reported in Fig. 9 implies $0.2 \leq P \leq 0.7$, which is totally in agreement with the variations observed in Fig. 10(c). In the case of simultaneous acquisition, the same pulse is used to acquire both images, and this provides a much more stable DOP estimation. It can be noticed that, in this case, the level of illumination has an influence on OSC estimation precision. A low illumination intensity actually leads directly to a low signal to noise ratio in intensity images, and it is shown in [31] that it decreases the performance of the OSC estimation. This is why low intensity pulses [the 26th, for example, in Fig. 10(d)] lead to relatively noisy OSC estimations, even in the case of simultaneous acquisition [see Fig. 10(c)]. However, these fluctuations are much lower than those observed with sequential acquisition.

\section{Conclusion}

We have described the design of a compact and efficient polarimetric imager based on a Wollaston polarization beam splitter and a single detector. This system is free from instrumental noise due to polarization switching elements and performs simultaneous acquisition of the two intensity images necessary to compute the OSC image. We have shown that its deformations can be calibrated and efficiently corrected by digital postprocessing. The estimation precision of the proposed system is thus mainly affected by detector noise and photon noise.

We have illustrated the advantage of simultaneous acquisition over a sequential one when the observed objects are moving, when the imaging medium is evolving because of turbulence, and when the intensity of light illumination is fluctuating. The two latter situations are of particular interest in active imaging. Indeed, the described system is well adapted to active imaging with narrow spectral width illumination. In order to address color or multispectral imaging, attention must be paid to the chromaticity of the device. An interesting perspective for future work would be to use this system in long range real conditions where pulsed illumination is required and where turbulence becomes significant. 
Arnaud Bénière's Ph.D. thesis is supported by the Délégation Générale pour l'Armement (DGA), Mission pour la Recherche et l'Innovation Scientifique (MRIS, contact: Jacques Blanc-Talon).

\section{References}

1. B. Laude-Boulesteix, A. Nazac, G. L. Naour, C. Genestie, L. Schwartz, B. Drevillon, and A. D. Martino, "Polarized images of the cervix," Proc. SPIE 5312, 243-246 (2004).

2. J. M. Bueno, J. Hunter, C. Cookson, M. Kisilak, and M. Campbell, "Improved scanning laser fundus imaging using polarimetry," J. Opt. Soc. Am. A 24, 1337-1348 (2007).

3. M. Alouini, F. Goudail, P. Réfrégier, A. Grisard, E. Lallier, and D. Dolfi, "Multispectral polarimetric imaging with coherent illumination: towards higher image contrast," Proc. SPIE 5432, 133-144 (2004).

4. S. Breugnot and P. Clémenceau, "Modeling and performances of a polarization active imager at $\lambda=806 \mathrm{~nm}$," Opt. Eng. 39, 2681-2688 (2000).

5. O. Emile, F. Bretenaker, and A. L. Floch, "Rotating polarization imaging in turbid media," Opt. Lett. 21, 1706-1708 (1996).

6. J. S. Tyo, M. P. Rowe, E. N. Pugh, and N. Engheta, "Target detection in optical scattering media by polarization-difference imaging," Appl. Opt. 35, 1855-1870 (1996).

7. Y. Y. Schechner, S. G. Narasimhan, and S. K. Nayar, "Polarization-based vision through haze," Appl. Opt. 42, 511-525 (2003).

8. S. Mujumdar and H. Ramachandran, "Imaging through turbid media using polarization modulation: dependence on scattering anisotropy," Opt. Commun. 241, 1-9 (2004).

9. F. Goudail and P. Réfrégier, "Statistical techniques for target detection in polarization diversity images," Opt. Lett. 26, 644-646 (2001)

10. O. Matoba and B. Javidi, "Three-dimensional polarimetric integral imaging," Opt. Lett. 29, 2375-2377 (2004).

11. B. Laude-Boulesteix, A. De Martino, B. Drévillon, and L. Schwartz, "Mueller polarimetric imaging system with liquid crystals," Appl. Opt. 43, 2824-2832 (2004).

12. F. Goudail, P. Terrier, Y. Takakura, L. Bigué, F. Galland, and V. Devlaminck, "Target detection with a liquid crystal-based passive Stokes polarimeter," Appl. Opt. 43, 274-282 (2004).

13. A. Jaulin, L. Bigue, and P. Ambs, "Implementation of a highspeed imaging polarimeter using a liquid crystal ferroelectric modulator," Proc. SPIE 6189, 618912 (2006).

14. M. Alouini, F. Goudail, A. Grisard, J. Bourderionnet, D. Dolfi, I. Baarstad, T. Løke, P. Kaspersen, and X. Normandin, "Active polarimetric and multispectral laboratory demonstrator: contrast enhancement for target detection," Proc. SPIE 6396, 63960B (2006).

15. P. Terrier and V. De Vlaminck, "Robust and accurate estimate of the orientation of partially polarized light from a camera sensor," Appl. Opt. 40, 5233-5239 (2001).
16. J. C. Ramella-Roman, K. Lee, S. A. Prahl, and S. L. Jacques, "Polarized light imaging with a handheld camera," Proc. SPIE 5068, 284-293 (2003).

17. A. L. Weijers, H. van Brug, and H. J. Frankena, "Polarization phase stepping with a Savart element," Appl. Opt. 37, 5150 5155 (1998)

18. M. C. Simon, "Wollaston prism with large split angle," Appl. Opt. 25, 369-376 (1986).

19. M. D. Perrin, J. R. Graham, and J. P. Lloyd, "The IRCAL polarimeter: design, calibration, and data reduction for an adaptive optics imaging polarimeter," Publ. Astron. Soc. Pac. 120, $555-570$ (2008).

20. A. R. Harvey, D. W. Fletcher-Holmes, A. Gorman, K. Altenbach, J. Arlt, and N. D. Read, "Spectral imaging in a snapshot," Proc. SPIE 5694, 110-119 (2005).

21. S. Huard, Polarization of Light (Wiley, 1997).

22. K. S. Kawabata, A. Okazaki, H. Akitaya, N. Hirakata, R. Hirata, Y. Ikeda, M. Kondoh, S. Masuda, and M. Seki, "A new spectropolarimeter at the Dodaira Observatory," Publ. Astron. Soc. Pac. 111, 898-908 (1999).

23. S. M. Kay, Fundamentals of Statistical Signal ProcessingVolume I: Estimation Theory (Prentice-Hall, 1993).

24. A. Bénière, F. Goudail, M. Alouini, and D. Dolfi, "Estimation precision of degree of polarization in the presence of signaldependent and additive Poisson noises," J. Eur. Opt. Soc. Rapid Publ. 3, 08002 (2008).

25. A. Bénière, F. Goudail, M. Alouini, and D. Dolfi, "Degree of polarization estimation in the presence of nonuniform illumination and additive Gaussian noise," J. Opt. Soc. Am. A 25, 919-929 (2008).

26. A. Bénière, F. Goudail, M. Alouini, and D. Dolfi, "Minimization of the influence of passive-light contribution in active imaging of the degree of polarization," Opt. Lett. 33, 2335-2337 (2008).

27. M. Alouini, F. Goudail, A. Grisard, J. Bourderionnet, D. Dolfi, A. Bénière, I. Baarstad, T. Løke, P. Kaspersen, X. Normandin, and G. Berginc, "Near-infrared active polarimetric and multispectral laboratory demonstrator for target detection," Appl. Opt. 48, 1610-1618 (2009).

28. L. Le Hors, P. Hartemann, D. Dolfi, and S. Breugnot, "Phenomenological model of paints for multispectral polarimetric imaging," Proc. SPIE 4370, 94-105 (2001).

29. M. Alouini, F. Goudail, N. Roux, L. Le Hors, P. Hartemann, S. Breugnot, and D. Dolfi, "Active spectro-polarimetric imaging: signature modeling, imaging demonstrator and target detection," Eur. Phys. J. Appl. Phys. 42, 129-139 (2008).

30. S. Sumrain and G. Giakos, "Sub pixel detection of polarimetric signatures of man-made targets," in Instrumentation and Measurement Technology Conference 2006 (IEEE, 2006), pp. $163-167$.

31. A. Bénière, F. Goudail, M. Alouini, and D. Dolfi, "Precision of degree of polarization estimation in the presence of additive Gaussian detector noise," Opt. Commun. 278, 264-269 (2007). 